\title{
The unreimbursed costs of preventing revision surgery in adult spinal deformity: analysis of cost-effectiveness of proximal junctional failure prevention with ligament augmentation
}

\author{
Michael M. Safaee, MD,, Cecilia L. Dalle Ore, BA, ${ }^{1}$ Corinna C. Zygourakis, MD, ${ }^{2}$ \\ Vedat Deviren, MD, ${ }^{3}$ and Christopher P. Ames, MD ${ }^{1,3}$ \\ Departments of ${ }^{1}$ Neurological Surgery and ${ }^{3}$ Orthopedic Surgery, University of California, San Francisco, California; and \\ 2Department of Neurological Surgery, Johns Hopkins Hospital, Baltimore, Maryland
}

OBJECTIVE Proximal junctional kyphosis (PJK) is a well-recognized complication of surgery for adult spinal deformity and is characterized by increased kyphosis at the upper instrumented vertebra (UIV). PJK prevention strategies have the potential to decrease morbidity and cost by reducing rates of proximal junctional failure (PJF), which the authors define as radiographic PJK plus clinical sequelae requiring revision surgery.

METHODS The authors performed an analysis of 195 consecutive patients with adult spinal deformity. Age, sex, levels fused, upper instrumented vertebra (UIV), use of 3-column osteotomy, pelvic fixation, and mean time to follow-up were collected. The authors also reviewed operative reports to assess for the use of surgical adjuncts targeted toward PJK prevention, including ligament augmentation, hook fixation, and vertebroplasty. The cost of surgery, including direct and total costs, was also assessed at index surgery and revision surgery. Only revision surgery for PJF was included.

RESULTS The mean age of the cohort was 64 years (range 25-84 years); 135 (69\%) patients were female. The mean number of levels fused was 10 (range 2-18) with the UIV as follows: 2 cervical (1\%), 73 upper thoracic (37\%), 108 lower thoracic (55\%), and 12 lumbar (6\%). Ligament augmentation was used in 99 cases (51\%), hook fixation in 60 cases (31\%), and vertebroplasty in 71 cases (36\%). PJF occurred in 18 cases (9\%). Univariate analysis found that ligament augmentation and hook fixation were associated with decreased rates of PJF. However, in a multivariate model that also incorporated age, sex, and UIV, only ligament augmentation maintained a significant association with PJF reduction (OR $0.196,95 \% \mathrm{Cl} 0.050-0.774 ; p=0.020$ ). Patients with ligament augmentation, compared with those without, had a higher cost of index surgery, but ligament augmentation was overall cost effective and produced significant cost savings. In sensitivity analyses in which we independently varied the reduction in PJF, cost of ligament augmentation, and cost of reoperation by $\pm 50 \%$, ligament augmentation remained a cost-effective strategy for PJF prevention.

CONCLUSIONS Prevention strategies for PJK/PJF are limited, and their cost-effectiveness has yet to be established. The authors present the results of 195 patients with adult spinal deformity and show that ligament augmentation is associated with significant reductions in PJF in both univariate and multivariate analyses, and that this intervention is costeffective. Future studies will need to determine if these clinical results are reproducible, but for high-risk cases, these data suggest an important role of ligament augmentation for PJF prevention and cost savings.

https://thejns.org/doi/abs/10.3171/2018.1.FOCUS17806

KEYWORDS spinal deformity; proximal junctional kyphosis; proximal junctional failure; cost-effectiveness

$\mathrm{P}$ ROXIMAL junctional kyphosis (PJK) is a well-recognized complication in patients undergoing surgical correction for adult spinal deformity. It presents with abnormal kyphosis immediately above the upper instrumented vertebra (UIV) and is quantified by measuring the sagittal Cobb angle between the inferior endplate of the UIV and the superior endplate of the second vertebral body above the UIV (UIV+2). The definition of PJK varies but is generally defined as an increase in kyphosis of more than $10^{\circ}-20^{\circ} .810,16 \mathrm{We}$ consider proximal junctional failure

ABBREVIATIONS BMP = bone morphogenetic protein; NNT = number needed to treat; PJF = proximal junctional failure; PJK = proximal junctional kyphosis; UIV = upper instrumented vertebra; UIV+1 = first vertebral body above the UIV; UIV+2 = second vertebral body above the UIV; UIV-1 = first vertebral body below the UIV. SUBMITTED January 2, 2018. ACCEPTED January 31, 2018. INCLUDE WHEN CITING DOI: 10.3171/2018.1.FOCUS17806. 
(PJF) to be radiographic evidence of PJK plus clinical sequelae that require revision surgery, including pain, neurological deficit, and impaired quality of life., 9,23

The causes of PJK/PJF are multifactorial and include age-related degeneration, poor bone quality, ligamentous fatigue, vertebral body fractures, instrumentation failure, and degenerative disc disease. . $, 2,8,23,26$ Additional surgical risk factors include older age at the time of surgery, ${ }^{3,13}$, 15,25 preoperative sagittal imbalance, ${ }^{16,17,19,20,30-32}$ increased curvature correction, ${ }^{16,17,30}$ and disruption of the facets and posterior ligamentous complex..$^{3,13,17,20}$ Rates of PJK vary by report and definition, but generally range from around $20 \%$ to $40 \%{ }^{8,14,15,24,31,32}$ Most cases occur relatively early in the postoperative period, with $66 \%$ of cases observed in the first 3 months and $80 \%$ within the first 18 months. ${ }^{30,32}$

Given the morbidity and cost associated with PJK/ PJF, particularly surgical revision, PJK prevention strategies are increasingly important. Surgical adjuncts, such as vertebroplasty, ligament augmentation, hook fixation at the UIV, and careful rod contouring, have been used with varying success. These adjuncts often go unreimbursed by insurance companies, despite their potential for significant cost savings. In this study, we sought to determine the efficacy of surgical adjuncts for PJK and model the costeffectiveness in a large population of patients with adult spinal deformity.

\section{Methods \\ Data Collection}

A series of 200 adult spinal deformity patients undergoing instrumented fusion were included in this study. Surgeries were performed from 2010 to 2017 at a single institution by 2 surgeons (V.D. and C.P.A). Patient demographics and surgical characteristics, including age, sex, indication for surgery, number of levels fused, use of bone morphogenetic protein (BMP), vertebroplasty (Cortoss cement, Stryker), hook fixation at the UIV (for constructs terminating in the upper thoracic spine), ligament augmentation (LigaPASS cable, Medicrea), 3-column osteotomy, and pelvic fixation, were collected.

Vertebroplasty, performed by tapping the cannulated pedicle screw trajectories and filling them with thrombincontaining hemostatic matrix to occlude venous channels, was done at the UIV and UIV+1 in a subset of lower-thoracic constructs. Cement was then injected into the vertebral bodies with a volume of $3 \mathrm{ml}$ at each entry site (6 $\mathrm{ml}$ per vertebral body). Ligament augmentation was performed in a subset of patients throughout the thoracic and lumbar spine at the UIV-1, UIV, and UIV+1 levels. This technique involves using a matchstick burr to drill through the center of the spinous processes at these levels and then passing a soft sublaminar cable through these holes in a mirrored fashion. The cable was pulled tight on each side by hand to allow testing of the exact tension desired and then fixed to the rod using supplied connectors. Spinous processes at these levels are loaded in slight extension to resist flexion at the terminal construct. Patients with neuromuscular or neurodegenerative disorders were excluded from analysis, and only patients with at least 6 months of follow-up were included. Only patients with data on total and direct cost of index and revision surgeries were included. PJF was defined as PJK of greater than $10^{\circ}$ plus clinical sequelae requiring revision surgery. Upper thoracic levels were defined as T1-6, and lower thoracic levels were defined as T7-12. All activities were approved by the Committee on Human Research, our institutional review board.

\section{Statistical Analysis}

Univariate analysis was performed using the chi-square test for categorical variables and the t-test for continuous variables. Binary logistic regression was used to generate a multivariate model that included all variables that were significant on univariate analysis plus those variables with known associations with PJF. Sensitivity analyses were performed by independently varying the cost of ligament augmentation, rate of PJF prevention, and cost of reoperation to determine if the intervention remained cost-effective under each of those scenarios. Statistical significance was defined as $\mathrm{p}<0.05$.

\section{Cost Analysis}

Total and direct hospital costs for each hospitalization were obtained from the hospital's accounting database and are reported in US dollars. Total costs include direct costs (e.g., imaging, medications, surgical supplies, hospital room) and indirect costs (such as administrative, facilities, and overhead fees not directly attributable to an individual patient). Of note, physician payments and noninstitutional costs incurred in the care of these patients (e.g., inpatient rehabilitation stay after discharge, outpatient physical therapy) are not available in our database and therefore not included in our calculations.

Cost-benefit analyses were performed by calculating the direct cost required for ligament augmentation in the index surgery versus the prevention of the cost of reoperation surgery. Sensitivity analyses were performed by independently varying the cost of ligament augmentation, rate of PJF prevention, and cost of reoperation by $\pm 50 \%$ to determine if the intervention remained cost-effective under each of those scenarios.

\section{Results}

\section{Patient Demographics}

A total of 200 patients were identified; 5 were excluded due to lack of cost data, leaving 195 patients in our final analysis. The mean age was 64 years (range 25-84 years), and 135 patients $(69 \%)$ were women. The mean number of levels fused was 10 (range 2-18). The UIV was cervical in 2 cases (1\%), upper thoracic in 73 cases (37\%), lower thoracic in 108 cases (55\%), and lumbar in 12 cases (6\%). Three-column osteotomies were used in 82 patients (42\%). Pelvic fixation was used in 190 cases (97\%). Surgical adjuncts were also quantified: ligament augmentation was used in 99 cases $(51 \%)$, hook fixation at the UIV in 60 cases $(31 \%)$, vertebroplasty in 71 cases (36\%), BMP in 133 cases $(68 \%)$, and 4-rod constructs with 3-column osteotomies in 78 cases (40\%). PJF occurred in 18 cases $(9 \%)$. The mean total cost of index surgery was $\$ 129,953 \pm$ $\$ 32,177$ (\$80,368 $\pm \$ 19,882$ in direct cost). The mean cost 
TABLE 1. Patient demographics and surgical characteristics

\begin{tabular}{|c|c|}
\hline Variable & Value \\
\hline No. of patients & 195 \\
\hline Mean age in yrs (range) & $64(25-84)$ \\
\hline \multicolumn{2}{|l|}{ Sex } \\
\hline Male & $60(31)$ \\
\hline Female & $135(69)$ \\
\hline \multicolumn{2}{|l|}{ Surgical characteristic } \\
\hline Mean levels fused (range) & $10(2-18)$ \\
\hline \multicolumn{2}{|l|}{ UIV } \\
\hline Cervical & $2(1)$ \\
\hline Upper thoracic & $73(37)$ \\
\hline Lower thoracic & $108(55)$ \\
\hline Lumbar & $12(6)$ \\
\hline $3 \mathrm{CO}$ & $82(42)$ \\
\hline Pelvic fixation & $190(97)$ \\
\hline \multicolumn{2}{|l|}{ Surgical adjuncts } \\
\hline Ligament augmentation & $99(51)$ \\
\hline Hook fixation at UIV & $60(31)$ \\
\hline Vertebroplasty & $71(36)$ \\
\hline BMP & $133(68)$ \\
\hline 4-rod construct w/ 3CO* & $78(40)$ \\
\hline PJF & $18(9)$ \\
\hline \multicolumn{2}{|l|}{ Mean cost } \\
\hline \multicolumn{2}{|l|}{ Primary surgery } \\
\hline Direct cost & $\$ 80,368 \pm 19,882$ \\
\hline Total cost & $\$ 129,953 \pm 32,177$ \\
\hline \multicolumn{2}{|l|}{ Revision surgery for PJF } \\
\hline Direct cost & $\$ 119,217 \pm 94,212$ \\
\hline Total cost & $\$ 193,277 \pm 152,613$ \\
\hline Mean FU duration in mos (range) & $22(6-78)$ \\
\hline
\end{tabular}

3CO = 3-column osteotomy; FU = follow-up.

Values are presented as the number of patients (\%) unless stated otherwise.

* The 4-rod construct was used in $40 \%$ of all patients and $95 \%$ of patients who underwent 3-column osteotomy (78 of 82).

of the revision surgery was $\$ 193,277 \pm \$ 152,613$ (\$119,217 $\pm \$ 94,212$ in direct cost). The mean length of follow-up was 22 months (range 6-78 months). These data are summarized in Table 1.

\section{Statistical Analysis of PJF Rates}

The effect of individual PJF prevention techniques was first assessed by univariate analysis. Ligament augmentation was associated with a $4 \%$ rate of PJF (4 of 99), compared with $15 \%$ (14 of 96) in patients without ligament augmentation $(\mathrm{p}=0.011)$. Hook fixation at the UIV was associated with a PJF rate of $2 \%$ (1 of 60), compared with $13 \%$ (17 of 135) in those without ( $p=0.014)$. Neither vertebroplasty nor BMP was associated with reduced rates of $\operatorname{PJF}(\mathrm{p}=0.819$ and $\mathrm{p}=0.498$, respectively). These data are summarized in Table 2.

Since hook fixation is only used in upper thoracic con-
TABLE 2. PJF rates with surgical adjuncts

\begin{tabular}{lccc}
\hline \multicolumn{1}{c}{ Surgical Adjunct } & $\begin{array}{c}\text { Rate of PJF w/ } \\
\text { Intervention }\end{array}$ & $\begin{array}{c}\text { Rate of PJF w/o } \\
\text { Intervention }\end{array}$ & p Value \\
\hline Ligament augmentation & $4 / 99(4)$ & $14 / 96(15)$ & 0.011 \\
\hline Hook fixation & $1 / 60(2)$ & $17 / 135(13)$ & 0.014 \\
\hline Vertebroplasty & $7 / 71(10)$ & $11 / 124(9)$ & 0.819 \\
\hline BMP & $11 / 133(8)$ & $7 / 62(11)$ & 0.498 \\
\hline
\end{tabular}

structs and vertebroplasty in lower thoracic constructs, we generated a multivariate model to control for other important confounders, including age, sex, and UIV, since hook fixation is only used in upper thoracic levels. In this multivariate model, only ligament augmentation was significantly associated with a reduction in PJF (OR 0.196, 95\% CI 0.050-0.774; $\mathrm{p}=0.020$ ). Age, sex, UIV, and hook fixation were not associated with a reduction in PJF. Results of multivariate analysis are summarized in Table 3.

Given the role of ligament augmentation in PJF prevention, we compared demographics, surgical characteristics, and both total and direct costs between patients who received ligament augmentation and those who did not. Age was significantly older in the ligament augmentation group (66 vs 63 years, $p=0.025$ ), as were the number of levels fused (11 vs 10 levels, $p=0.043$ ). The UIV did not significantly differ with respect to upper and lower thoracic constructs; however, there were more lumbar UIVs in the group without ligament augmentation $(11 \%$ vs $1 \%, \mathrm{p}=$ 0.002 ). We compared the UIV in patients with and without ligament augmentation who developed PJF. In the group with ligament augmentation, 4 patients developed PJF. The UIV levels in these patients were as follows: 1 at C-7 and 3 at $\mathrm{T}-10$. In the group without ligament augmentation, the UIV levels were as follows: 3 each at T-10 and T-12, 2 each at T-11 and L-1, and 1 each at T-3, T-4, T-9, and L-2.

Rates of PJF were lower in the ligament augmentation group ( $4 \%$ vs $15 \%, \mathrm{p}=0.011$ ), but the mean follow-up was also shorter in the ligament augmentation group (16 vs 29 months, $\mathrm{p}<0.001)$. The cost of index surgery was significantly higher for patients with ligament augmentation, and the cost of revision surgery for patients with ligament augmentation was notably lower; however, the difference did not reach statistical significance. These data are summarized in Table 4.

TABLE 3. Multivariate analysis of variables associated with PJF

\begin{tabular}{llc}
\hline \multicolumn{1}{c}{ Variable } & \multicolumn{1}{c}{ OR $(95 \% \mathrm{Cl})$} & Significance $(\mathrm{p}$ value $)$ \\
\hline Age & $1.038(0.979-1.100)$ & 0.216 \\
\hline Female sex & $2.301(0.603-8.780)$ & 0.223 \\
\hline UIV & & \\
\hline Upper thoracic & 1.000 (reference) & 0.365 \\
\hline Lower thoracic & $3.217(0.467-22.157)$ & 0.235 \\
\hline Lumbar & $5.096(0.526-49.363)$ & 0.160 \\
\hline Ligament augmentation & $0.196(0.050-0.774)$ & 0.020 \\
\hline Hook fixation & $0.544(0.034-5.894)$ & 0.544 \\
\hline
\end{tabular}


TABLE 4. Comparison of patients with and without ligament augmentation

\begin{tabular}{|c|c|c|c|}
\hline Variable & w/ Ligament Augmentation $(n=99)$ & w/o Ligament Augmentation $(n=96)$ & $\mathrm{p}$ Value \\
\hline Mean age in yrs & 66 & 63 & 0.025 \\
\hline Female sex & $67(68)$ & $68(71)$ & 0.633 \\
\hline \multicolumn{4}{|l|}{ Surgical features } \\
\hline Mean no. of levels fused & 11 & 10 & 0.043 \\
\hline \multicolumn{4}{|l|}{ UIV } \\
\hline Cervical & $1(1)$ & $1(1)$ & 0.983 \\
\hline Upper thoracic & $42(42)$ & $31(32)$ & 0.144 \\
\hline Lower thoracic & $55(56)$ & $53(55)$ & 0.961 \\
\hline Lumbar & $1(1)$ & $11(11)$ & 0.002 \\
\hline Hook fixation & $42(42)$ & $18(19)$ & $<0.001$ \\
\hline Vertebroplasty & $47(47)$ & $24(25)$ & 0.001 \\
\hline $3 \mathrm{CO}$ & $39(39)$ & $43(45)$ & 0.445 \\
\hline Pelvic fixation & $97(98)$ & $93(97)$ & 0.626 \\
\hline PJF & $4(4)$ & $14(15)$ & 0.011 \\
\hline \multicolumn{4}{|l|}{ Mean cost } \\
\hline \multicolumn{4}{|l|}{ Primary op } \\
\hline Direct cost & $\$ 85,449 \pm 17,504$ & $\$ 75,128 \pm 20,893$ & $<0.001$ \\
\hline Total cost & $\$ 139,131 \pm 27,842$ & $\$ 120,488 \pm 33,711$ & $<0.001$ \\
\hline \multicolumn{4}{|l|}{ Reop } \\
\hline Direct cost & $\$ 74,586 \pm 23,606$ & $\$ 131,968 \pm 103,395$ & 0.296 \\
\hline Total cost & $\$ 122,661 \pm 41,246$ & $\$ 213,453 \pm 167,607$ & 0.308 \\
\hline
\end{tabular}

Values are presented as the number of patients (\%) unless stated otherwise.

\section{Cost-Benefit Analysis}

We then performed a cost-benefit analysis for ligament augmentation in the prevention of PJF. Specifically, we compared the cost of ligament augmentation in the index case $(\$ 1100)$ versus the cost of reoperation for PJF $(\$ 119,217)$. Since ligament augmentation produced a $10.5 \%$ reduction in the rate of PJF in our patient cohort, we calculated that 9.5 patients had to receive ligament augmentation in their index surgery in order to prevent 1 reoperation for PJF (this is the number needed to treat [NNT]). This means that we spent $\$ 10,450$ for ligament augmentation upfront in 9.5 patients in order to prevent a $\$ 119,217$ revision surgery in 1 patient. Scaled to a theoretical cohort of 100 patients receiving ligament augmentation, the estimated savings would be $\$ 1,144,916$.

To assess the robustness and generalizability of our results, we performed sensitivity analyses for the cost-benefit of ligament augmentation for PJF prevention. The value of each parameter (PJF reduction rate, cost of ligament augmentation, and cost of reoperation) was independently varied by $\pm 50 \%$. At baseline, $\$ 108,767$ was saved by using ligament augmentation upfront to prevent reoperation for PJF. The use of ligament augmentation remained cost-effective, with savings per 100 patients ranging from $\$ 515,889$ to $\$ 1,772,374$, in all variations. These data are summarized in Table 5.

Given that different hospitals have various implant prices, we performed a continuous sensitivity analysis for ligament augmentation implant price (Fig. 1). The threshold price under which ligament augmentation remained cost-effective in preventing PJF was $\$ 12,549$, which is a significant increase from the price currently negotiated at our center (\$1100) and more than the cost difference between patients with and without ligament augmentation $(\$ 11,035)$.

\section{Discussion}

Prevention strategies for PJK/PJF are of increasing importance in adult spinal deformity. In addition to the significant morbidity and clinical impact on patients, the cost of revision surgery carries a significant economic impact. ${ }^{18}$ Reducing rates of PJF therefore has the potential to produce significant cost savings. ${ }^{29}$ Several techniques believed to reduce the risk of PJK/PJF include extending fusion constructs to include levels with baseline segmental kyphosis of more than $5^{\circ}$, using composite metals with greater flexibility, minimizing the destruction of soft tissue at the UIV, achieving optimal spinal balance postoperatively, and using transition rods. ., $^{2,5,8,19,20}$ The use of hook fixation at the UIV, as shown in our data, is also associated with decreased rates of PJK/PJF. This technique provides a theoretical advantage since the hooks require less violation of the facet joint and provide more dynamic fixation at the top of the construct. ${ }^{10,12,16}$ Several studies have compared PJK rates in patients with spinal hooks to those with pedicle screws at the UIV; rates of PJK in those with hooks ranged from $0 \%$ to $30 \%$, compared with $30 \%$ to $35 \%$ in those with pedicle screw constructs. ${ }^{10,16}$

Our clinical practice has evolved over the past 5 years, 
TABLE 5. Sensitivity analysis for cost-benefit analysis of ligament augmentation for PJF prevention (direct costs)

\begin{tabular}{|c|c|c|c|c|c|}
\hline Parameter & Cost of Reop & NNT & Cost of LA & Cost to Prevent Single PJF & Cost Savings w/ LA Per 100 Pts \\
\hline Base case & $\$ 119,217$ & 9.5 & $\$ 1,100$ & $\$ 10,450$ & $\$ 1,144,916$ \\
\hline \multicolumn{6}{|c|}{ Varied parameters } \\
\hline \multicolumn{6}{|c|}{ Reduction in PJF } \\
\hline $5.25 \%$ & $\$ 119,217$ & 19.0 & $\$ 1,100$ & $\$ 20,952$ & $\$ 515,889$ \\
\hline $15.75 \%$ & $\$ 119,217$ & 6.3 & $\$ 1,100$ & $\$ 6,984$ & $\$ 1,767,668$ \\
\hline \multicolumn{6}{|l|}{ Cost of LA } \\
\hline$\$ 550$ & $\$ 119,217$ & 9.5 & $\$ 550$ & $\$ 5,225$ & $\$ 1,199,916$ \\
\hline$\$ 1650$ & $\$ 119,217$ & 9.5 & $\$ 1,650$ & $\$ 15,675$ & $\$ 1,089,916$ \\
\hline \multicolumn{6}{|l|}{ Cost of reop } \\
\hline$\$ 59,609$ & $\$ 59,609$ & 9.5 & $\$ 1,100$ & $\$ 10,450$ & $\$ 517,458$ \\
\hline$\$ 178,826$ & $\$ 178,826$ & 9.5 & $\$ 1,100$ & $\$ 10,450$ & $\$ 1,772,374$ \\
\hline
\end{tabular}

$\mathrm{LA}=$ ligament augmentation; $\mathrm{pts}=$ patients.

with PJF prevention becoming a significant priority for all deformity cases. ${ }^{28}$ Since the proposed mechanism of PJK in the upper thoracic spine is ligamentous fatigue, we use hook fixation at the UIV for all constructs terminating at those levels. We also introduce terminal kyphosis into the rod so it is never forced into place, which prevents additional loading forces to the construct and minimizes the risk for screw pullout or junctional stress. For constructs terminating in the lower thoracic spine, we perform vertebroplasty at the UIV and UIV-1 levels since failures at the thoracolumbar spine are often due to fractures. We use ligament augmentation in all cases, as it is an emerging strategy for PJF prevention. The goal of this technique is to reinforce the junction between the UIV and adjacent noninstrumented vertebrae while minimizing facet joint violation. ${ }^{27}$ Our data indicate that this strategy is highly effective in reducing the rate of PJF after surgery for adult spinal deformity, producing a $10.5 \%$ reduction in PJF in patients with ligament augmentation compared with those without ligament augmentation.

Prior studies have demonstrated that readmissions and reoperations in patients with adult spinal deformity are associated with a significant increase in costs and reduction in the cost-effectiveness of deformity correction. ${ }^{6,22,29}$ Reoperations for PJF often require large surgeries with extension of instrumentation, making PJF a particularly costly complication. In this context, our analysis suggests that the use of ligament augmentation is highly cost-effective. Implant costs associated with ligament augmentation total approximately $\$ 1100$, or $1.4 \%$ of the direct cost of the primary surgery. Given the NNT of 9.5 , the cost of preventing 1 reoperation is $\$ 10,450$, which is $\$ 108,767$ less than the mean cost of a reoperation for PJF. The potential cost savings are thus substantial. Recent estimates suggest that rates of patients undergoing fusion for adult spinal deformity are increasing, with 6728 cases in 2013 at a rate

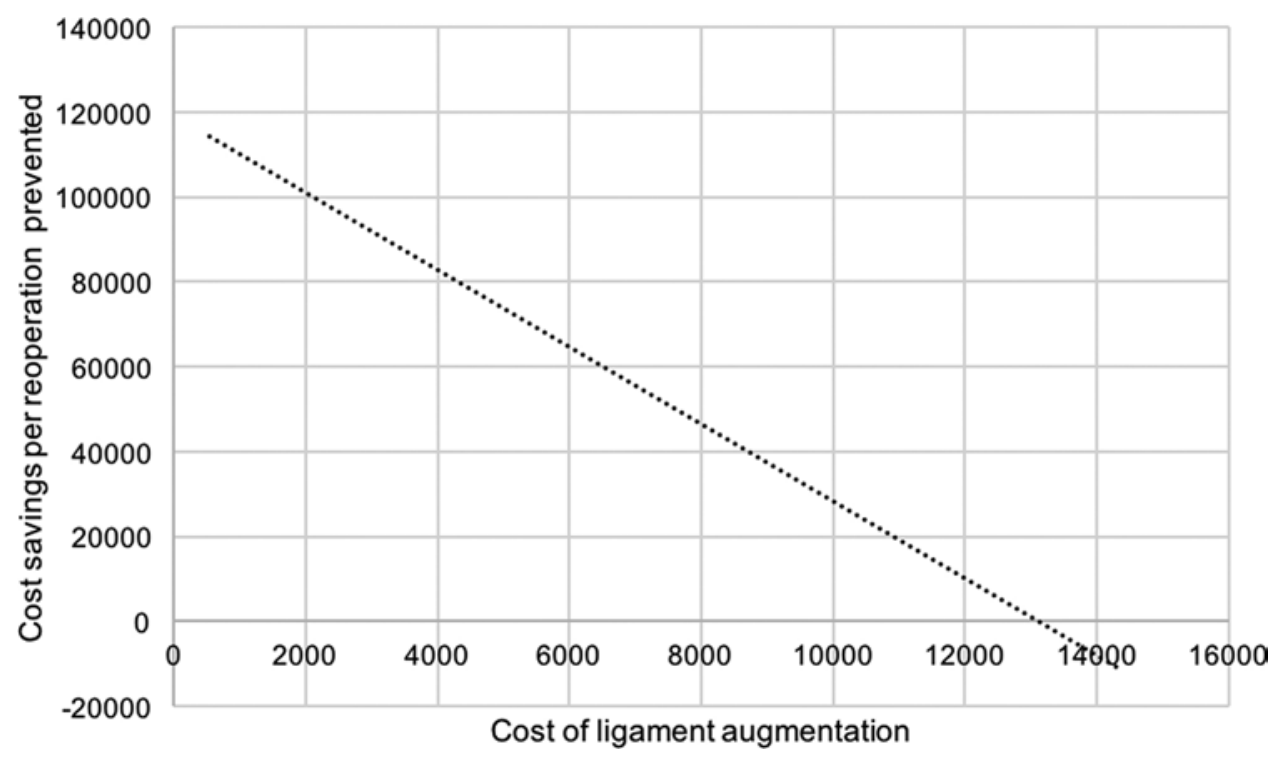

FIG. 1. Sensitivity analysis of ligament augmentation price. When varying the price of ligament augmentation, the intervention remains cost-effective as long as the price is less than $\$ 12,549$. The current negotiated price at our center is $\$ 1100$. 
of 13.9 per 100,000 adults per year in the United States. ${ }^{33}$ Based on an NNT of 9.5, ligament augmentation has the potential to prevent 10.5 reoperations per 100 patients with a savings of over $\$ 1.25$ million. When scaled to 6728 cases annually, this results in a savings of over $\$ 84$ million per year. Although not all of these cases are large deformity corrections that are generalizable to the population included in this study, even a conservative estimate using one-third of all deformity corrections (approximately 2241 patients per year), would result in a savings of over $\$ 28$ million annually by using ligament augmentation in primary index surgeries for deformity.

Direct costs associated with the primary surgery, ligament augmentation, and reoperation for PJF are likely to vary by center based on negotiated prices with implant manufacturers and other determinants of surgical cost, including patient factors, surgeon preferences, hospital practices, and regional variability. ${ }_{6,7,11,21,22}$ Along similar lines, a relative paucity of data exists regarding the reduction in PJF produced by ligament augmentation, ${ }^{27}$ and it is possible that the reduction in PJF achieved with ligament augmentation may vary as this technique is adopted at other centers. Our sensitivity analysis indicates that even if ligament augmentation produced half the reduction in PJF we observed at our institution, it would still be highly cost-effective in these settings.

There are certain limitations to the present study that are inherent to its retrospective design, such as selection bias and moderate sample size. Although this was a cohort of purely adult spinal deformity patients, they were not randomized to PJF prevention strategy or surgical plan. The retrospective design also limits the ability to control for confounding variables such as bone mineral density scores or other clinical factors that can contribute to increased risk of PJF. This study design also limits our ability to capture postoperative events, including revision surgery, since patients were not included in a prospective cohort where adverse events or revision surgeries are more completely captured. These cohorts have some inherent heterogeneity since ligament augmentation is a more recent technique. Our practice pattern has evolved in a fluid manner, so that patients who underwent ligament augmentation were also more likely to have hook fixation (for constructs terminating in the upper thoracic spine), vertebroplasty (for constructs terminating in the lower thoracic spine or thoracolumbar junction), BMP, and gentle contouring of the rods, which could not be retrospectively assessed. The use of these additional surgical adjuncts helps explain the nearly $\$ 10,000$ difference in the direct cost of primary surgery in the ligament augmentation group compared with the group without ligament augmentation, since the price of ligament augmentation alone is only $\$ 1100$. We utilized a multivariate model to help control for these differences, but future studies should utilize an entirely contemporary cohort across multiple centers to determine the reproducibility of these data. In addition, it is important to note that our cost data do not include outpatient costs, such as physical therapy or rehabilitation. We would expect that PJF reoperation costs also including outpatient costs would be even higher than those calculated here, making ligament augmentation even more cost-effective for these patients.

\section{Conclusions}

Ligament augmentation is a novel strategy for PJK/PJF prevention. Even when controlling for age, sex, UIV, and use of other surgical adjuncts, ligament augmentation continues to demonstrate a significant reduction in PJF and the need for revision surgery. Using sensitivity analysis, we showed that even dramatic variations in the reduction of PJF rates, cost of ligament augmentation, and cost of reoperation do not change the significant cost-effectiveness of this treatment. Future studies will need to determine if these data are reproducible in large cohorts of adult spinal deformity patients and control for other important confounders including bone mineral density. However, given the current cost of ligament augmentation and relative ease of implementing this strategy in the operating room, this technique warrants consideration for all adult spinal deformity cases, particularly for patients at high risk for PJK/ PJF.

\section{References}

1. Anderson AL, McIff TE, Asher MA, Burton DC, Glattes RC: The effect of posterior thoracic spine anatomical structures on motion segment flexion stiffness. Spine (Phila Pa 1976) 34:441-446, 2009

2. Arlet V, Aebi M: Junctional spinal disorders in operated adult spinal deformities: present understanding and future perspectives. Eur Spine J 22 (Suppl 2):S276-S295, 2013

3. Bridwell KH, Lenke LG, Cho SK, Pahys JM, Zebala LP, Dorward IG, et al: Proximal junctional kyphosis in primary adult deformity surgery: evaluation of 20 degrees as a critical angle. Neurosurgery 72:899-906, 2013

4. Cammarata M, Aubin CE, Wang X, Mac-Thiong JM: Biomechanical risk factors for proximal junctional kyphosis: a detailed numerical analysis of surgical instrumentation variables. Spine (Phila Pa 1976) 39:E500-E507, 2014

5. Denis F, Sun EC, Winter RB: Incidence and risk factors for proximal and distal junctional kyphosis following surgical treatment for Scheuermann kyphosis: minimum five-year follow-up. Spine (Phila Pa 1976) 34:E729-E734, 2009

6. Fischer CR, Terran J, Lonner B, McHugh B, Warren D, Glassman S, et al: Factors predicting cost-effectiveness of adult spinal deformity surgery at 2 years. Spine Deform 2:415-422, 2014

7. Glassman SD, Berven S, Kostuik J, Dimar JR, Horton WC, Bridwell K: Nonsurgical resource utilization in adult spinal deformity. Spine (Phila Pa 1976) 31:941-947, 2006

8. Glattes RC, Bridwell KH, Lenke LG, Kim YJ, Rinella A, Edwards C II: Proximal junctional kyphosis in adult spinal deformity following long instrumented posterior spinal fusion: incidence, outcomes, and risk factor analysis. Spine (Phila Pa 1976) 30:1643-1649, 2005

9. Hart RA, McCarthy I, Ames CP, Shaffrey CI, Hamilton DK, Hostin R: Proximal junctional kyphosis and proximal junctional failure. Neurosurg Clin N Am 24:213-218, 2013

10. Helgeson MD, Shah SA, Newton PO, Clements DH III, Betz RR, Marks MC, et al: Evaluation of proximal junctional kyphosis in adolescent idiopathic scoliosis following pedicle screw, hook, or hybrid instrumentation. Spine (Phila Pa 1976) 35:177-181, 2010

11. Hostin R, Robinson C, O’Brien M, Ames C, Schwab F, Smith JS, et al: A multicenter comparison of inpatient resource use for adult spinal deformity surgery. Spine (Phila Pa 1976) 41:603-609, 2016

12. Kim HJ, Bridwell KH, Lenke LG, Park MS, Ahmad A, Song $\mathrm{KS}$, et al: Proximal junctional kyphosis results in inferior 
SRS pain subscores in adult deformity patients. Spine (Phila Pa 1976) 38:896-901, 2013

13. Kim HJ, Bridwell KH, Lenke LG, Park MS, Song KS, Piyaskulkaew C, et al: Patients with proximal junctional kyphosis requiring revision surgery have higher postoperative lumbar lordosis and larger sagittal balance corrections. Spine (Phila Pa 1976) 39:E576-E580, 2014

14. Kim HJ, Lenke LG, Shaffrey CI, Van Alstyne EM, Skelly AC: Proximal junctional kyphosis as a distinct form of adjacent segment pathology after spinal deformity surgery: a systematic review. Spine (Phila Pa 1976) 37 (22 Suppl):S144S164, 2012

15. Kim YJ, Bridwell KH, Lenke LG, Glattes CR, Rhim S, Cheh G: Proximal junctional kyphosis in adult spinal deformity after segmental posterior spinal instrumentation and fusion: minimum five-year follow-up. Spine (Phila Pa 1976) 33:2179-2184, 2008

16. Kim YJ, Bridwell KH, Lenke LG, Kim J, Cho SK: Proximal junctional kyphosis in adolescent idiopathic scoliosis following segmental posterior spinal instrumentation and fusion: minimum 5-year follow-up. Spine (Phila Pa 1976) 30:20452050, 2005

17. Kim YJ, Lenke LG, Bridwell KH, Kim J, Cho SK, Cheh G, et al: Proximal junctional kyphosis in adolescent idiopathic scoliosis after 3 different types of posterior segmental spinal instrumentation and fusions: incidence and risk factor analysis of 410 cases. Spine (Phila Pa 1976) 32:2731-2738, 2007

18. Lau D, Funao H, Clark AJ, Nicholls F, Smith J, Bess S, et al: The clinical correlation of the Hart-ISSG proximal junctional kyphosis severity scale with health-related quality-of-life outcomes and need for revision surgery. Spine (Phila Pa 1976) 41:213-223, 2016

19. Lee GA, Betz RR, Clements DH III, Huss GK: Proximal kyphosis after posterior spinal fusion in patients with idiopathic scoliosis. Spine (Phila Pa 1976) 24:795-799, 1999

20. Maruo K, Ha Y, Inoue S, Samuel S, Okada E, Hu SS, et al: Predictive factors for proximal junctional kyphosis in long fusions to the sacrum in adult spinal deformity. Spine (Phila Pa 1976) 38:E1469-E1476, 2013

21. McCarthy I, O’Brien M, Ames C, Robinson C, Errico T, Polly DW Jr, et al: Incremental cost-effectiveness of adult spinal deformity surgery: observed quality-adjusted life years with surgery compared with predicted quality-adjusted life years without surgery. Neurosurg Focus 36(5):E3, 2014

22. McCarthy IM, Hostin RA, Ames CP, Kim HJ, Smith JS, Boachie-Adjei O, et al: Total hospital costs of surgical treatment for adult spinal deformity: an extended follow-up study. Spine J 14:2326-2333, 2014

23. McClendon J Jr, O'Shaughnessy BA, Sugrue PA, Neal CJ, Acosta FL Jr, Koski TR, et al: Techniques for operative correction of proximal junctional kyphosis of the upper thoracic spine. Spine (Phila Pa 1976) 37:292-303, 2012

24. Mendoza-Lattes S, Ries Z, Gao Y, Weinstein SL: Proximal junctional kyphosis in adult reconstructive spine surgery results from incomplete restoration of the lumbar lordosis relative to the magnitude of the thoracic kyphosis. Iowa Orthop J 31:199-206, 2011

25. O'Leary PT, Bridwell KH, Lenke LG, Good CR, Pichelmann MA, Buchowski JM, et al: Risk factors and outcomes for catastrophic failures at the top of long pedicle screw constructs: a matched cohort analysis performed at a single center. Spine (Phila Pa 1976) 34:2134-2139, 2009
26. Oxland TR, Panjabi MM, Southern EP, Duranceau JS: An anatomic basis for spinal instability: a porcine trauma model. J Orthop Res 9:452-462, 1991

27. Safaee MM, Deverin V, Dalle Ore C, Scheer JK, Lau D, Osorio JA, et al: Ligament augmentation for prevention of proximal junctional kyphosis and proximal junctional failure in adult spinal deformity. J Neurosurg Spine [epub ahead of print February 23, 2018; DOI: 10.3171/2017.9.SPINE1710]

28. Safaee MM, Osorio JA, Verma K, Bess S, Shaffrey CI, Smith JS, et al: Proximal junctional kyphosis prevention strategies: a video technique guide. Oper Neurosurg (Hagerstown) 13:581-585, 2017

29. Theologis AA, Miller L, Callahan M, Lau D, Zygourakis C, Scheer JK, et al: economic impact of revision surgery for proximal junctional failure after adult spinal deformity surgery: a cost analysis of 57 operations in a 10-year experience at a major deformity center. Spine (Phila Pa 1976) 41:E964E972, 2016

30. Wang J, Zhao Y, Shen B, Wang C, Li M: Risk factor analysis of proximal junctional kyphosis after posterior fusion in patients with idiopathic scoliosis. Injury 41:415-420, 2010

31. Yagi M, Akilah KB, Boachie-Adjei O: Incidence, risk factors and classification of proximal junctional kyphosis: surgical outcomes review of adult idiopathic scoliosis. Spine (Phila Pa 1976) 36:E60-E68, 2011

32. Yagi M, King AB, Boachie-Adjei O: Incidence, risk factors, and natural course of proximal junctional kyphosis: surgical outcomes review of adult idiopathic scoliosis. Minimum 5 years of follow-up. Spine (Phila Pa 1976) 37:1479-1489, 2012

33. Zygourakis CC, Liu CY, Keefe M, Moriates C, Ratliff J, Dudley RA, et al: Analysis of national rates, cost, and sources of cost variation in adult spinal deformity. Neurosurgery 82:378-387, 2018

\section{Disclosures}

Dr. Zygourakis: travel grants to attend resident education courses from Globus, NuVasive, DePuy, and AOSpine. Dr. Deviren: consultant for NuVasive, Guidepoint, Biomet, Medicrea, and Pfizer; and royalties from NuVasive. Dr. Ames: employee of UCSF; consultant for K2M, Medicrea, DePuy Synthes, Medtronic, and Stryker; and royalties from NuVasive, Biomet Spine, Stryker, and DePuy Synthes.

\section{Author Contributions}

Conception and design: Deviren, Ames. Acquisition of data: Safaee, Dalle Ore, Zygourakis. Analysis and interpretation of data: Safaee, Dalle Ore, Zygourakis, Ames. Drafting the article: Safaee, Dalle Ore. Critically revising the article: Safaee, Dalle Ore, Zygourakis, Ames. Reviewed submitted version of manuscript: Safaee, Dalle Ore, Ames. Approved the final version of the manuscript on behalf of all authors: Safaee. Statistical analysis: Safaee, Dalle Ore. Administrative/technical/material support: Deviren, Ames.

\section{Correspondence}

Michael Safaee: University of California, San Francisco, CA. michael.safaee@ucsf.edu. 